\title{
Effects of center of mass kinematics on ball velocity during jump throwing in handball
}

\begin{abstract}
The purpose of the present study was for investigating the relationships between body center of mass kinematic parameters and ball velocity during jump throwing in handball. Ten Handball players participated in this study; eight synchronized highspeed cameras were used at a $100 \mathrm{~Hz}$ frequency. SIMI 3D motion analyses system was used for capture and analyses, and SPSS statistical software was used to examine the relationships between COM kinematic parameters and ball velocity. The results indicated that the cocking phase and COM kinematical parameters are very important when evaluating jump throwing. In addition, the horizontal jump more associated with ball velocity than a vertical jump, although many situations in the game required the vertical jump. Thus, we recommend the coaches to focus to apply these conclusions to training programs, and for evaluating the performance level of handball players.
\end{abstract}

Volume 2 Issue 3 - 2018

\author{
Abdel-Rahman Ibrahim Akl, Mona Hamdy \\ Salem \\ Alexandria University, Egypt
}

Correspondence: Abdel Rahman IA, Faculty of Physical Education (Abo Qir), Alexandria University,Alexandria, Egypt, Email abdelrahman.akl@alexu.edu.eg

Received: May 18, 2018 | Published: June 21, 2018

Keywords: biomechanics, 3D kinematic analysis, jump throwing

\section{Introduction}

In handball competition, the jump throw is the most used throw at the goal. Previous studies analyzed the performances of team handball players in a different aspect and concluded that angular kinematical parameters contributed significantly to differences in ball release velocity during throws and focused on upper limb angular kinematics when analysis jump throwing in handball. ${ }^{1-5}$ And several studies emphasized the effects of aerobic fatigue, analysis the relation between throwing velocity and throwing accuracy, muscle power, and factors Influencing on ball throwing velocity in handball. ${ }^{6,7}$ Several studies suggest that jump height for throws is considered one of the important parameters in team handball. A high jump results a good position to throw over the block of the rival defender and associated by playing positions. Especially, when players shoot from the backcourt position or have more time for a shot and when attempting to react to goalkeeper actions. ${ }^{8,9}$ In our study, we focused on examining the center of mass (COM) kinematics and its relationship with ball velocity, The COM is a virtual point, which the total body mass can be concentrated and related to human balance and stability. Center of mass variables are becoming a great interest in sports to determine the level of performance in many sports activity like running, football, ${ }^{10}$, ${ }^{11}$ volleyball,,${ }^{12}$ judo, ${ }^{13}$ long jump. ${ }^{14}$ On the other hand, the center of mass excursions yield the best conditions for mastering balance and keeping the lower body more stable and effective.

Many methods have been proposed to assess the position of COM, several studies are based on kinematic models of the whole human body and are known as segmental or kinematical methods. Other studies focus on the displacement of trunk or of the pelvis, there are called sacrum or reconstructed pelvis methods. ${ }^{4}$ Segmental method regards at the body structure as a set of rigid bodies, identified by anatomical landmarks. Their positions in time and space are detected using motion capture systems. ${ }^{15}$ additionally, the maximum change in COM velocity is considered as an important parameter that is potentially related to performance..$^{15}$ In context, the velocity considers a determinant of throwing performance in handball. Therefore, the purpose of the present study was investigating the relationships between body center of mass kinematic parameters and ball velocity during jump throwing in handball.

\section{Methods}

Ten Handball players participated in this study (age: $20.8 \pm 1.21$ years; body mass: $82.8 \pm 8.57 \mathrm{~kg}$; height: $189.6 \pm 8.65 \mathrm{~cm}$ ). After 15-minute warm-up, the participants completed jump-throws from the 7-m penalty line after three running steps. A total of five successful attempts were recorded for each participant, one-minute rest between attempts. The best attempt was selected for 3D linear kinematic analysis. The kinematic variables of center of body mass (displacement, velocity, and acceleration) on the three dimensions and resultant, and ball velocity were measured using a 3-D motion capture system (Simi Reality motion analysis V. 9.0.6. Eight synchronized Basler (scA640-120gc) High-Speed Cameras were used at a $100 \mathrm{~Hz}$ frequency) that tracked the position of the reflective markers on anatomical landmarks according Hanavan model. The 3D coordinates were the X (medio-lateral), Y (anterior-posterior) and Z (vertical) directions respectively. The subjects performed the jump shot attempts on a strain-gage force platform (MP4060®, Bertec Corporation, Columbus, $\mathrm{OH}$, USA), which measured the ground reaction force (GRF), the data were sampled using an analog-to-digital converter at a rate of $1000 \mathrm{~Hz}$, an $\mathrm{x}, \mathrm{y}, \mathrm{z}$ orthogonal coordinate system was used to describe the forces (F) relative to the force plate. In addition, the sign convention designated vertical upward $(\mathrm{Fz})$, lateral $(\mathrm{Fx})$, and anterior (Fy) motions. The jump throwing skill was split into three phases according the arm movement (Cocking phase, Acceleration phase, and Following phase). The three phases include selected eight moments (Initial contact (IC), Maximum ground reaction force (Max-GRF), Initial flight to take-off (IF), Maximum arm back cocking (Max-BC), Maximum height of center of mass (Max-CGH), Maximum height of throwing arm (Max-HH), Ball release (RB), and Landing (GC)) during performance. To determine the relationships between COM kinematic parameters and ball velocity during throwing performance, these variables were correlated with the ball velocity using IBM SPSS statistics V. 22 software. 


\section{Results and discussion}

This study was carried out to investigate the relationships between body center of mass kinematic parameters and ball velocity during jump throwing in handball. The results indicated that the ball velocity was $(21.82 \pm 0.75 \mathrm{~m} / \mathrm{s})$ and the performance was examined according three phases (Cocking phase, Acceleration phase, and Following phase (Figure 1). Including eight moments; initial contact, maximum Ground Reaction force, initial flight, maximum back cocking, maximum height of body CG during flight, maximum height of hand throwing CG during flight, release ball, and ground contact. (Table 1 )indicated that a significant correlation between ball velocity and COM displacement $\mathrm{Y}$ during the moments; initial flight: $\mathrm{p}<0.05$, maximum height of body $C G$ during flight: $p<0.01$, maximum height of hand throwing CG during flight: $p<0.05$, release ball: $p<0.05$, and ground contact: $\mathrm{p}<0.01$ ). In addition, a significant correlation between ball velocity and $\mathrm{COM}$ displacement $\mathrm{R}$ during the moments; initial flight: $\mathrm{p}<0.05$, maximum height of body $\mathrm{CG}$ during flight: $\mathrm{p}<0.05$, maximum height of hand throwing CG during flight: $\mathrm{p}<0.01$, release ball: $\mathrm{p}<0.01$, and ground contact: $\mathrm{p}<0.01 .^{16}$

Table I Correlation between Body COM kinematical parameters and ball velocity during Jump throwing phases in Handball

\begin{tabular}{|c|c|c|c|c|c|c|c|c|c|}
\hline \multirow[b]{2}{*}{ Parameters(unit) } & \multirow[b]{2}{*}{ coordinates } & \multicolumn{3}{|c|}{ Cocking phase } & \multicolumn{3}{|c|}{ Acceleration phase } & \multicolumn{2}{|c|}{ Following phase } \\
\hline & & IC & Max-GRF & IF & Max-BC & $\begin{array}{l}\text { Max- } \\
\text { CGH }\end{array}$ & $\begin{array}{l}\text { Max- } \\
\text { HH }\end{array}$ & RB & GC \\
\hline \multirow{4}{*}{ Displacement(m) } & $x$ & .131 & -.006 & -.034 & -.030 & -.099 & -.019 & -.027 & -.058 \\
\hline & $y$ & -.173 & .489 & $.710 *$ & .524 & $.823 * *$ & $.738 *$ & $.762 *$ & $.854 * *$ \\
\hline & $z$ & .190 & -.472 & -.099 & -.004 & .039 & .295 & .226 & $-.886-* *$ \\
\hline & $\mathrm{R}$ & .220 & -.582 & $-.636-*$ & .309 & $.646^{*}$ & $.796 * *$ & $.812^{* *}$ & $.826 * *$ \\
\hline \multirow{4}{*}{ Velocity(m/s) } & $x$ & -.204 & -.231 & -.077 & -.081 & .019 & .330 & -.287 & -.102 \\
\hline & $y$ & $.680 *$ & $.776 * *$ & $.838 * *$ & .807 & $.892 * *$ & $.656 *$ & .588 & .550 \\
\hline & $\mathrm{z}$ & -.538 & -.289 & .127 & .542 & -.018 & .492 & -.034 & -.367 \\
\hline & $\mathrm{R}$ & $.691 *$ & $.72 I^{*}$ & $.864 * *$ & $.786 * *$ & $.899 * *$ & .512 & $.734^{*}$ & $.696 *$ \\
\hline \multirow{4}{*}{$\begin{array}{l}\text { Acceleration } \\
\left(\mathrm{m} / \mathrm{s}^{2}\right)\end{array}$} & $x$ & .038 & .143 & -.403 & .170 & -.125 & -.555 & -.399 & .186 \\
\hline & $y$ & .176 & .126 & .120 & .311 & .044 & -.358 & .086 & -.016 \\
\hline & z & -.339 & -.108 & -.034 & .502 & .003 & -.105 & -.038 & $.652 *$ \\
\hline & $\mathrm{R}$ & .074 & -.116 & .105 & -.454 & .021 & .210 & .152 & .108 \\
\hline
\end{tabular}

Note: Coordinates. X (medio-lateral), Y (anterior-posterior), Z (vertical), and R (resultant); Moments. IC, initial contact (end of last step); max-GRF, maximum Ground Reaction force; IF, initial flight; max-BC, maximum back cocking; max-CGH, maximum height of body CG during flight; max-HH, maximum height of hand throwing CG during flight; RB, release ball; GC, ground contact; *. Correlation is significant at $\mathrm{p}<0.05$; $* *$. Correlation is significant at $\mathrm{p}<0.0 \mathrm{I}$.
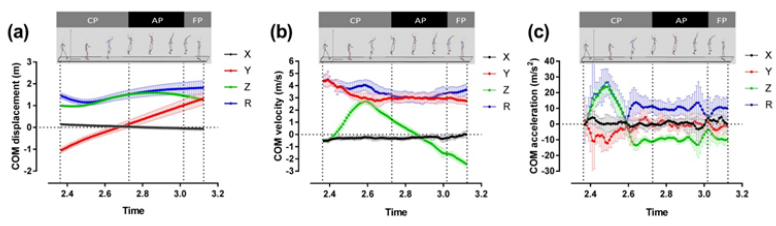

Figure I Body COM kinematical parameters during Jump throwing phases: (A) displacement (X, Y, Z, R); (B) velocity (X, Y, Z, R); (C) acceleration (X, Y, $\mathrm{Z}, \mathrm{R})$.

\section{Note: CP (Cocking phase); AP (Acceleration phase); FP (Following phase).}

The major finding of Table 1 results was the relationship between displacement $\mathrm{Y}$ with ball velocity at initial flight (take-off) and emphasized the importance of this moment when evaluating jump throwing, and it can be considered a discriminate moment of jump throwing. Moreover, initial flight moment affected on next moments (Table 1). As well as, the results indicated that a significant correlation between ball velocity and $\mathrm{COM}$ velocity $\mathrm{Y}$ during the moments; initial contact: $\mathrm{p}<0.05$, maximum Ground Reaction force: $\mathrm{p}<0.01$, initial flight: $p<0.01$, maximum back cocking: $p<0.01$, maximum height of body CG during flight: $\mathrm{p}<0.01$, and maximum height of hand throwing $C G$ during flight: $\mathrm{p}<0.05$. In addition, a significant correlation between ball velocity and COM velocity $\mathrm{R}$; initial contact: $\mathrm{p}<0.05$, maximum Ground Reaction force: $\mathrm{p}<0.05$, initial flight: $\mathrm{p}<0.01$, maximum back cocking: $\mathrm{p}<0.01$, maximum height of body CG during flight: $\mathrm{p}<0.01$, release ball: $\mathrm{p}<0.05$, and ground contact: $\mathrm{p}<0.05$. In addition, a significant correlation between ball velocity and COM acceleration $\mathrm{Z}$ (ground contact: $\mathrm{p}<0.05$ ). Consequently, COM velocity $\mathrm{Y}$ is one of the important parameters during jump throwing due the gained velocity from run-up (Figure 1b), it is influencing on the performance from initial contact to the second moment before ball release. In addition, it affected on the resultant of $\mathrm{COM}$ velocity during the performance. Interestingly, the results showed non-significant between COM accelerations and ball velocity, except vertical velocity when ground contact after landing may because the gravity.

\section{Conclusion}

Our findings indicated that COM kinematical parameters are very important when evaluating jump-throwing performance in handball, especially displacement and velocity on movement direction (anterior-posterior). Furthermore, the cocking phase is very important as a preparation phase to acquire the velocity of the body and arm throwing. Finally, the horizontal jump more associated with ball velocity than a vertical jump, although many situations in the game required the vertical jump. Thus, we recommend the coaches to focus to apply these conclusions for training programs, and for evaluating the performance level of handball players.

\section{Acknowledgments}

None.

\section{Conflict of interest}

Author declares that there is no Conflict of interest.

\section{References}

1. Serrien B, Clijsen R, Blondeel J, et al. Differences in Ball Speed and Three-Dimensional Kinematics between Male and Female Handball Players During a Standing Throw with Run-Up. BMC Sports Sci Med Rehabil. 2015;7:27. 
2. Van den Tillaar R, Ettema G. A Three-Dimensional Analysis of Overarm Throwing in Experienced Handball Players. $J$ Appl Biomech. 2007;23(1):12-9.

3. Wagner H, Buchecker M, Von Duvillard SP, et al. Kinematic Description of Elite Vs. Low Level Players in Team-Handball Jump Throw. J Sports Sci Med. 2010;9(1):15-23.

4. Wagner H, Pfusterschmied J, Tilp M, et al. Upper-Body Kinematics in Team-Handball Throw, Tennis Serve, and Volleyball Spike. Scand J Med Sci Sports. 2014;24(2):345-54

5. Wagner H, Pfusterschmied J, Von Duvillard SP, et al. Performance and Kinematics of Various Throwing Techniques in Team-Handball. J Sports Sci Med. 2011;10(1):73-80.

6. Antonio García J, Sabido R, Barbado D, et al. Analysis of the Relation between Throwing Speed and Throwing Accuracy in Team-Handball According to Instruction. European Journal of Sport Science. 2013;13(2):149-54.

7. Plummer HA, Dawn Oliver G. The Effects of Aerobic Fatigue on Jump Shot Kinematics in Team Handball Players. Journal of Biomedical Engineering and Informatics. 2016. 2(1):65-75.

8. Ravier G, Demouge J. Comparison of Lower Limb Strength Characteristics between Youth and Adult Elite Female Team Handball Players. Science \& Sports. 2016;31(3):e39-e46.

9. Wagner H, Finkenzeller T, Wurth S, et al. Individual and Team Performance in Team-Handball: A Review. J Sports Sci Med. 2014;13(4):808-16.
10. Haran F, Tierney R, Wright W, et al. No Acute Changes in Postural Control after Soccer Heading. Br J Sports Med. 2004;38(5): 561-67.

11. Manolopoulos E, Papadopoulos C, Kellis E. Effects of Combined Strength and Kick Coordination Training on Soccer Kick Biomechanics in Amateur Players. Scand J Med Sci Sports. 2006;16(2):102-10.

12. Wagner H, Tilp M, Von Duvillard SP, et al. Kinematic Analysis of Volleyball Spike Jump. Int J Sports Med. 2009;30(10):760-5.

13. Imamura RT, Hreljac A, Escamilla RF, et al. A Three-Dimensional Analysis of the Center of Mass for Three Different Judo Throwing Techniques. $J$ Sports Sci Med. 2006;5:122-31.

14. Hay JG, Nohara H. Techniques Used by Elite Long Jumpers in Preparation for Takeoff, Journal of Biomechanics, 1990;23(3): 229-39.

15. Mapelli, Zago M, Fusini L, et al. Validation of a Protocol for the Estimation of Three-Dimensional Body Center of Mass Kinematics in Sport. Gait Posture. 2014;39(1):460-5.

16. Akl A-R, Hassan A. Identifying the Role of Cocking Phase During Jump Throwing in Handball Using 3d Kinematic Analyses', in 21st annual Congress of the European College of Sport Science (ECSS). Vienna, Austria: European College of Sport Science; 2016. 